\title{
Site-specific tagging proteins with a rigid, small and stable transition metal chelator, 8- hydroxyquinoline, for paramagnetic NMR analysis
}

Yin Yang ${ }^{1}$, Feng Huang ${ }^{1}$, Thomas Huber ${ }^{2}$ and Xun-Cheng Su${ }^{1}$

1. State Key Laboratory of Elemento-Organic Chemistry, College of Chemistry,

Collaborative Innovation Center of Chemical Science and Engineering (Tianjin)

Nankai University, Tianjin 300071, China

2. Research School of Chemistry, Australian National University,

Canberra ACT 0200, Australia

* Corresponding author

phone: +86 2223500623

e-mail: xunchengsu@nankai.edu.cn

Keywords: 8-hydroxyquinoline, protein structure, paramagnetic NMR spectroscopy, pseudocontact shift, paramagnetic relaxation enhancement

\begin{abstract}
Design of a paramagnetic metal binding motif in a protein is a valuable way of understanding the function, dynamics and interactions of a protein by paramagnetic NMR spectroscopy. Several strategies have been proposed to sitespecifically tag proteins with paramagnetic lanthanide ions. Here, we report a simple approach of engineering a transition metal binding motif via site-specific labelling of a protein with 2-vinyl-8-hydroxyquinoline (2V-8HQ). The protein-2V-8HQ adduct forms a stable complex with transition metal ions, $\mathrm{Mn}(\mathrm{II}), \mathrm{Co}(\mathrm{II}), \mathrm{Ni}(\mathrm{II}), \mathrm{Cu}(\mathrm{II})$ and Zn(II). The paramagnetic effects generated by these transition metal ions were evaluated by NMR spectroscopy. We show that 2V-8HQ is a rigid and stable transition metal binding tag. The coordination of the metal ion can be assisted by protein sidechains. More importantly, tunable paramagnetic tensors are simply obtained in an $\alpha$-helix that possesses solvent exposed residues in positions $i$ and $i+3$, where $i$ is the residue position to be mutated to cysteine and $\mathrm{i}+3$ is Gln or Glu or i-4 is His. The coordination of a sidechain carboxylate or imidazole to cobalt (II) results in different structural geometries, leading to different paramagnetic tensors as shown by experimental data.
\end{abstract}




\section{Introduction}

One of the mainstream uses of transition metals in structural biology is exploiting the diverse paramagnetic properties of these ions (Bertini and Luchinat 1996; Bertini and Luchinat 1999; Clore and Iwahara 2009) by high resolution NMR spectroscopy. The intrinsic paramagnetic property of transition metal ions provides valuable structural restraints in biomolecules, which are governed by exact theory (Abragam and Bleaney 1970; Bertini and Luchinat 1996). These paramagnetic effects include paramagnetic relaxation enhancement (PRE), pseudocontact shifts (PCSs) and residual dipolar couplings (RDCs), and are experimentally observable by NMR spectroscopy. In particular, PCSs are the chemical shift differences between the diamagnetic and a paramagnetic species, and thus can be measured with high accuracy. While PRE only depends on the distance between the paramagnetic ion and the observed nuclear spin, PCS contains both distance and direction information of nuclear spins with respect to the paramagnetic center (Bertini et al 2002; Clore and Iwahara 2009; Otting 2010). Because of their high chemical similarity and the large variation of paramagnetism, lanthanide ions have received increasing interests in structural biology by means of high resolution NMR spectroscopy (Geraldes 1993; Bertini et al 2002; Graldes and Luchinat 2003; Otting 2008; Bertini 2008; Otting 2010; Pintacuda et al 2007; Liu et al 2014). As many proteins do not contain a paramagnetic center, strategies in site-specific labeling of proteins with paramagnetic species have been reviewed (Rodriguez-Castañeda et al 2006; Su and Otting 2010; Koehler and Meiler 2011; Liu et al 2014). Paramagnetic transition metal ions have an even longer history in paramagnetic protein NMR spectroscopy (La Mar et al 1970; Bertini and Luchinat 1984; Bertini et al 1993; Bertini and Luchinat 1996; Donaire et al 1998; Bertini et al 2005; Arnesano et al 2005), however, site-specific labeling of biomolecules with a rigid transition metal ion is less studied (Gochin 1998; Man et al 2010; Nguyen et al 2011). The complex of high-spin Co(II) produces the largest paramagnetic anisotropy, Mn(II) complex has strong PRE effect on nuclear spins because it has five unpaired electrons and usually longer relaxation time (Bertini and Luchinat 1996), and Cu(II) ion forms most stable complex but produces generally weaker PREs than $\mathrm{Mn}(\mathrm{II})$. In the case of $\mathrm{Ni}(\mathrm{II})$ ion, its paramagnetic effects are largely determined by its coordination with the chelating ligand. In comparison with lanthanide ions, transition metal ions have fewer coordination sites (generally up to six) and prefer to coordinate with histidine sidechain. This offers additional advantages in designing smaller and more rigid paramagnetic tags, as the stability and rigidity of the paramagnetic tag strongly influences the interpretation of paramagnetic restraints from NMR spectroscopy.

8-Hydroxyquinoline (8HQ), a frequently used organic chelator for metal ions, forms stable complexes with transition metal ions in varying molar ratio (Johnston and Freiser 1952). Incorporation of 8HQ into a protein has been recently reported via bioorthogonal protein synthesis (Lee et al 2009; Liu et al 2013; Park et al 2015). 8HQ is 
a bidenate ligand and has only two coordinating atoms. Therefore, one 8HQ alone is not able to immobilize the metal ion without additional coordination by the protein, and the trend of aggregation due to the unsaturated coordination sites of the metal ion should be prevented in the design of paramagnetic tags for NMR or other spectroscopic analysis in structural biology. Additional intramolecular coordination with protein sidechains have been shown to avert taginduced protein aggregation and also significantly restrict the metal ion, thus suppressing the positional fluctuations of the paramagnetic center (Su et al 2008; Swarbrick et al 2011; Yagi et al 2013; Huang et al 2013).

The high reactivity and chemoselectiviy of a 4-vinyl pyridine moiety to thiol groups of proteins has been demonstrated in protein modifications for paramagnetic NMR and EPR analysis (Li et al 2012; Yang et al 2013; Qi et al 2014). Here we report an 8HQ derivative, 2-vinyl-8-hydroxyquinoline (2V-8HQ), which can be site-specifically attached to a protein via a Michael addition-like thiol-ene reaction (Fig 1 and Scheme 1). To test the reactivity of 2V-8HQ to protein thiols, we made two single-point cysteine mutants of human ubiquitin, T22C and A28C, respectively. The combination of a small functional tag and protein sidechain in immobilizing the transition metal ions was analyzed. The rigidity of the transition metal ion with respect to the protein and the affinity of sidechains of glutamate and histidine for the metal ion were evaluated by high resolution NMR spectroscopy and isothermal titration calorimetry (ITC). In the structure of ubiquitin, the solvent exposed sidechains of residues Glu24 and Asp32 are close to Thr22 and Ala28, respectively, and are possible to bind metal ion in addition to 8HQ. As imidazole is a preferred ligand for transition metal ions, the double-point mutant E24H/A28C was constructed. The PCSs of A28C2V-8HQ and E24H/A28C-2V-8HQ complexed with Co(II) differed significantly, suggesting different paramagnetic tensors. Tunable paramagnetic anisotropy is highly valuable in structural biology, and we show that different sets of paramagnetic susceptibility tensors can simply be obtained by the changing the metal ion coordination by straightforward protein sidechain mutagenesis.

\section{Materials and methods}

Protein expression and purification

The target proteins were expressed using optimized high-density methods (Marley et al 2001; Cao et al 2014) and typically $20 \mathrm{mg}{ }^{15} \mathrm{~N}$-protein was obtained from $250 \mathrm{~mL}$ M9 media.

Synthesis of 2V-8HQ 


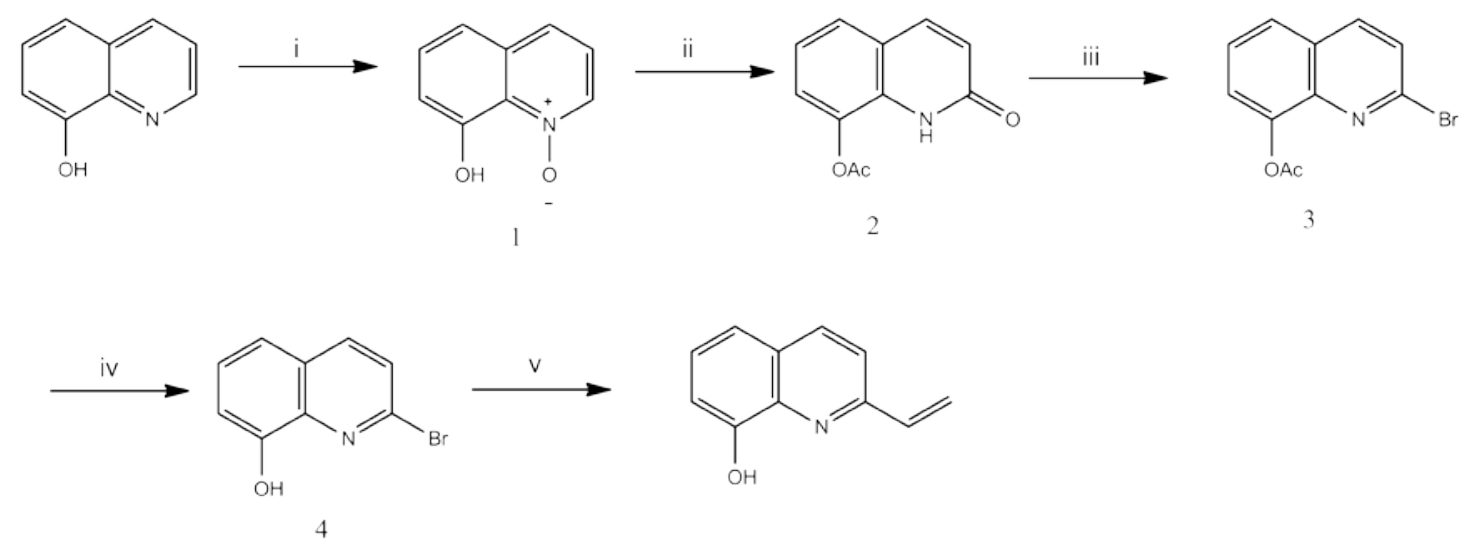

Scheme 1. Reagents and conditions: (i) m-CPBA/DCM; (ii) $\mathrm{Ac}_{2} \mathrm{O} / \mathrm{Ar} / 100{ }^{\circ} \mathrm{C}$; (iii) $\mathrm{POBr}_{3} / \mathrm{Ar} / \mathrm{CHCl}_{3}$; (iv) basic hydrolysis; (v) Triethoxyvinylsilane/Pd(OAc) $2 / \mathrm{TBAF} / \mathrm{PPh}_{3} / \mathrm{DMF}$

N-oxide-8-hydroxyquinoline (1): $0.5 \mathrm{~g}$ 8-Hydroxyquinoline was dissolved in $4 \mathrm{~mL}$ dichloromethane and $0.69 \mathrm{~g}$ meta-chloroperbenzoic acid (m-CPBA) was added stepwise under ice-cooled water bath. The resulting mixture was stirred at room temperature overnight. $7.14 \mathrm{~mL} 2 \mathrm{M} \mathrm{NaOH}$ was added slowly to the above solution. The organic layer was separated and the water phase was washed with dichloromethane. The organic fractions were combined and washed with brine, dried with sodium sulfate, and then filtered. The organic solvent was removed under reduced pressure, resulting in $0.39 \mathrm{~g}$ the title compound as yellow solid (yield, $70.3 \%{ }^{1} \mathrm{H} \mathrm{NMR}\left(300 \mathrm{MHz}, \mathrm{CDCl}_{3}\right) \delta 8.25$ (dd, J= 6.0, 0.8 Hz, 1H), 7.80 (d, J= 8.1 Hz, 1H), 7.50 (t, J= $8.0 \mathrm{~Hz}, 1 \mathrm{H}), 7.25-7.21$ (m, 2H), 7.08 (dd, J= 7.9, 1.1 $\mathrm{Hz}, 1 \mathrm{H})$

Compounds 2, 3 and 4 were synthesized according the published methods with minor modifications (Petitjean et al 2005).

2-Vinyl-8-hydroxyquinoline (2V-8HQ): Similar to the previously reported coupling reaction (Alacid and Najera 2008), the mixture of $1.2 \mathrm{~g}(0.03 \mathrm{~mol}) \mathrm{NaOH}, 20 \mathrm{mg}(0.09 \mathrm{mmol}) \mathrm{Pd}(\mathrm{OAc})_{2}, 2.28 \mathrm{~g}(0.01 \mathrm{~mol})$ compound 4, 2.53 $\mathrm{mL}(0.012 \mathrm{mmol})$ triethoxyvinylsilane, $30 \mathrm{~mL} \mathrm{H} \mathrm{H}_{2} \mathrm{O}$ and 30 g PEG 2000 was stirred at $80-90{ }^{\circ} \mathrm{C}$ for $10 \mathrm{~h}$ and the resulting mixture was extracted with diethyl ether. The organic phase was combined and washed with brine. The organic solution was dried with sodium sulfate, and filtered. The organic solvent was removed under reduced pressure, resulting $2.0 \mathrm{~g}$ title compound as white powder (yield, $88.9 \%) .{ }^{1} \mathrm{H}$ NMR $\left({ }^{1} \mathrm{H} 400 \mathrm{MHz}, \mathrm{CDCl}_{3}\right) \delta 7.95(\mathrm{~d}$, $\mathrm{J}=8.6 \mathrm{~Hz}, 1 \mathrm{H}), 7.43(\mathrm{~d}, \mathrm{~J}=8.6 \mathrm{~Hz}, 1 \mathrm{H}), 7.27$ (q, J= $8.3 \mathrm{~Hz}, 1 \mathrm{H}), 7.15$ (d, J= $8.2 \mathrm{~Hz}, 1 \mathrm{H}), 7.05$ (d, J= $7.5 \mathrm{~Hz}, 1 \mathrm{H})$, 6.86 (dd, J= 17.7, $10.9 \mathrm{~Hz}, 1 \mathrm{H}), 6.18$ (d, J=17.7 Hz, 1H), 5.52 (d, J= 10.9 Hz, 1H). 
All NMR spectra of protein samples were recorded at $298 \mathrm{~K}$ on a Bruker AV600 NMR spectrometer equipped with a QCI-cryoprobe. The protein samples were in $20 \mathrm{mM}$ 2-(N-morpholino)ethanesulfonic acid (MES) buffer at pH 6.4 unless otherwise indicated. The recycle delay for PRE measurements was $2 \mathrm{~s}$ for both diamagnetic and paramagnetic samples.

Site-specific labeling of ubiquitin mutant with $2 \mathrm{~V}-8 \mathrm{HQ}$

$0.5 \mathrm{mM}$ solution of ${ }^{15} \mathrm{~N}$-labeled protein in $20 \mathrm{mM}$ tris(hydroxymethyl)aminomethane (Tris) pH 7.8 was first mixed with $0.2 \mathrm{mM}$ tris(2-carboxyethyl)phosphine (TCEP), and then five equivalents of 2V-8HQ (100 mM in ethanol stock solution) were added to the protein solution. The $\mathrm{pH}$ was adjusted to 7.8 using $1.0 \mathrm{M} \mathrm{NaOH}$ and the mixture was incubated at room temperature for $10 \mathrm{~h}$. The ligation product was purified by gel filtration. The overall yield of purified ligation product was approximately $80 \%$.

\section{Results and Discussion}

$2 \mathrm{~V}-8 \mathrm{HQ}$ is a thiol-specific reaction reagent for labeling proteins

The ligation reaction was monitored by recording ${ }^{15} \mathrm{~N}-\mathrm{HSQC}$ spectra of the reaction mixture with the ${ }^{15} \mathrm{~N}$ ubiquitin mutant protein with 2V-8HQ. Quantitative ligation of protein and 2V-8HQ was achieved by incubating the protein with five equivalents of tag at room temperature, $\mathrm{pH} 7.8$ in $20 \mathrm{mM}$ Tris buffer for $10 \mathrm{~h}$. The reaction of 2V8HQ with the ubiquitin mutants T22C, A28C and E24H/A28C was completed within 10 hours at pH 7.8 in $20 \mathrm{mM}$ Tris buffer (monitored by ${ }^{15} \mathrm{~N}-\mathrm{HSQC}$ spectra), and the reactivity of 2V-8HQ towards free thiols is significantly higher than the previously reported 4V-PyMTA (Yang et al 2013) and also 4V-DPA (Li et al 2012). The tagging reaction rate increases with increasing $\mathrm{pH}$ and the ligation can be completed within five hours at $\mathrm{pH} 8.5$.

Mass analysis by MALDI-TOF mass spectrometry confirmed that proteins were conjugated with only one tag (Fig S2), suggesting no additional groups including amino group of the N-terminal methionine and amino groups of lysine sidechains reacted with $2 \mathrm{~V}-8 \mathrm{HQ}$. This is consistent with NMR chemical shift mapping in ${ }^{15} \mathrm{~N}-\mathrm{HSQC}$ spectra between free protein and its 2V-8HQ conjugates (Fig. 2 and Fig. S1), as the chemical shift perturbations were mainly located around the ligation site of protein thiol. The high specificity of the reactions of 2V-8HQ with protein thiols suggests that this small tag can be applied to label proteins via the formation of a stable thiolether tether.

Interaction of 2V-8HQ protein adducts with transition metal ions

The interactions of transition metal ions with ubiquitin-2V-8HQ adducts were first analyzed by titration of 
Co(II) into the solution of protein conjugates, because Co(II) has the largest magnetic anisotropy among transition metal ions and has been shown previously to be an excellent metal probe in metalloproteins (Bertini and Luchinat 1984). Addition of $\mathrm{CoCl}_{2}$ into the solution of $0.1 \mathrm{mM}{ }^{15} \mathrm{~N}-\mathrm{A} 28 \mathrm{C}-2 \mathrm{~V}-8 \mathrm{HQ}$ generated a new set of signals in the ${ }^{15} \mathrm{~N}-$ HSQC spectrum with increasing intensities as the concentration of cobalt ion increases. The association-dissociation between the metal bound and unbound protein complexes is in slow exchange on the NMR time scale, indicating a tight binding. Only one paramagnetic species was observed during the titration, corresponding to the complex formed by protein-2V-8HQ and Co(II) ion. Similarly, only one paramagnetic species was observed for the T22C-2V-8HQ and E24H/A28C-2V-8HQ adducts with Co(II), respectively. The A28C-2V-8HQ and E24H/A28C-2V-8HQ adducts complexed with Co(II) experienced significantly larger chemical shift perturbations than T22C-2V-8HQ (Fig 3 and Fig S3), and therefore our analyses mainly focus on the A28C-2V-8HQ and E24H/A28C-2V-HQ conjugates in the following study. The slow exchange between Mn(II), Co(II), Ni(II), Cu(II) and Zn(II) complexed ubiquitin-2V-8HQ was also observed in ${ }^{15} \mathrm{~N}-\mathrm{HSQC}$ spectra.

Bing affinity measurement of ubiquitin-2V-8HQ with transition metal ions

The association constants between transition metal ion and protein-2V-8HQ are difficult to measure by NMR due to a slow exchange between ion bound and unbound state of the protein, and the thermodynamic stabilities of protein-2V-8HQ complexed with transition metal ion were analyzed by isothermal titration calorimetry (ITC) (Fig S4). In $20 \mathrm{mM}$ MES buffer at $\mathrm{pH}$ 6.4, the binding constants of Co(II) and Ni(II) with protein-2V-8HQ were determined by ITC measurement. The titration curves for the metal ions $\mathrm{Mn}(\mathrm{II}), \mathrm{Cu}(\mathrm{II})$ and $\mathrm{Zn}$ (II) to protein failed to produce the binding constant under identical conditions used with $\mathrm{Co}(\mathrm{II})$ and $\mathrm{Ni}(\mathrm{II})$. The interactions of $\mathrm{Mn}(\mathrm{II})$, Zn(II) and $\mathrm{Cu}(\mathrm{II})$ ions with protein-2V-8HQ did not result in a sigmoidal titration curve. Despite the fact that A28C2V-8HQ and E24H/A28C-2V-8HQ show similar slow exchange between free protein and metal bound complex, they differ greatly in binding affinity with $\mathrm{Co}(\mathrm{II})$ and $\mathrm{Ni}(\mathrm{II})$ ions. The determined association constants, $\mathrm{Ka}$, of E24H/A28C-2V-8HQ for Ni(II) and Co(II) are $4.8 \times 10^{5}$ and $4.0 \times 10^{5} \mathrm{M}^{-1}$, respectively, which are ten times of those of A28C-2V-8HQ $\left(6.7 \times 10^{4} \mathrm{M}^{-1}\right.$ with $\mathrm{Ni}(\mathrm{II})$ and $4.9 \times 10^{4} \mathrm{M}^{-1} \mathrm{M}^{-1}$ with Co(II)). The change of Glu 24 to His 24 in the ubiquitin-2V-8HQ adducts increases the binding affinity for the transition metal ions almost an order, supporting that the imidazole group is a preferred ligand over carboxylate in a protein. Paramagnetic anisotropy of Co(II) complexed with ubiquitin-2V-8HQ

The paramagnetic properties of transition metal ions bound to protein-2V-8HQ conjugates were analyzed with high resolution NMR spectroscopy. PCSs were determined by the chemical shift differences between the paramagnetic and diamagnetic species (Fig. 3, Fig. S3 and Fig. S5), using Zn(II) as the diamagnetic references. Ni(II) 
has been extensively applied in structural biology due to its anisotropic paramagnetic properties (Jensen and Led 2006; Jensen et al 2004), however, its complex with protein-2V-8HQ produced only very small chemical shift changes in the present study. As expected, the NMR resonances were also much less attenuated by PREs compared with $\mathrm{Co}(\mathrm{II}), \mathrm{Mn}(\mathrm{II})$ and $\mathrm{Cu}(\mathrm{II})$ because most cross-peaks were observable in ${ }^{15} \mathrm{~N}-\mathrm{HSQC}$ spectra even for residues close to the ligation site (Fig S5). The negligible chemical shift changes and lack of cross-peak attenuation observed with $\mathrm{Ni}(\mathrm{II})$ in the ubiquitin-2V-8HQ conjugates is likely due to the formation of a square-planar complex which is diamagnetic because the electrons occupying the $3 \mathrm{~d}$ orbitals are fully paired. The diamagnetic properties of the protein-2V-8HQ-Ni(II) complexes were similar to that of a previously published histidine-coordinated protein complex (Jones et al 2005).

Fig 3 shows the sizable PCSs induced by the Co(II) complex which produces larger chemical shift changes (up to $0.8 \mathrm{ppm}$ ) than any of the other transition metal ions. Similarly, a large number of residues are observable in the ${ }^{15} \mathrm{~N}$-HSQC spectrum of the ubiquitin-2V-8HQ complexed with other metal ions. In the case of the copper (II) complex, proton spins within $10 \AA$ of the paramagnetic center are generally inaccessible to NMR measurement (Arnesano et al 2003), nonetheless a large number of residues are observable in ${ }^{15} \mathrm{~N}-\mathrm{HSQC}$ spectra of the ubiquitin2V-8HQ adducts (Fig. 4). In the case of Mn(II), which causes stronger PRE than $\mathrm{Cu}(\mathrm{II})$, several peaks in the ${ }^{15} \mathrm{~N}$ HSQC spectra of ubiquitin-2V-8HQ Mn(II) complex display line broadening, yet the same signals as with the copper complex can be clearly observed.

The paramagnetic anisotropy was analyzed for the protein complexes of Co(II) as the PCSs produced by $\mathrm{Cu}(\mathrm{II})$ are rather small. As shown in Fig 3B, the T22C-2V-8HQ conjugate in complex with cobalt (II) generated smaller PCSs than those of the A28C-2V-8HQ and E24H/A28C-2V-8HQ conjugates. The different sizes of PCSs in the three conjugates indicate different degrees of averaging. Since mobility of either the coordination to the paramagnetic center or of the tether linking 2V-8HQ and protein will result in paramagnetic averaging, the sizes of the PCSs and RDCs are sensitive to this flexibility. In the structure of ubiquitin, Thr22 resides at the start of the first $\alpha$-helix and Ala28 is in the middle of the same helix. Smaller PCSs in the T22C-2V-8HQ-Co(II) complex indicate a stronger averaging of paramagnetic effects due to the flexibility of the coordination to the cobalt ion, implying that coordination with the protein sidechain probably does not sufficiently restrict the paramagnetic center in this situation.

In general, the PCS of a nuclear spin is described by equation 1 ,

$$
P C S=\frac{1}{12 \pi r^{3}}\left[\Delta \chi_{a x}\left(3 \cos ^{2} \theta-1\right)+1.5 \Delta \chi_{r h} \sin ^{2} \theta \cos 2 \phi\right]
$$


where $r, \theta$, and $\phi$ are the polar coordinates of the nuclear spin relative to the principal axes of the magnetic susceptibility anisotropy tensor, and $\Delta \chi_{\mathrm{ax}}$ and $\Delta \chi_{\mathrm{rh}}$ the axial and rhombic components of the $\Delta \chi$-tensor, respectively. As shown in equation (1), it is evident that reliable tensors can be determined provide that paramagnetic center was accurately defined. Using the program Numbat (Schmitz et al 2008), the magnetic susceptibility tensor was determined by fitting the PCS data of backbone amide protons to the crystal structure of ubiquitin (Ramage et al 1994) and RDC refined NMR structure (Maltsev et al 2014), respectively. Both structures gave similar results (Table 1). Excellent correlations between the experimental PCS and back-calculated data are observed (Fig S6), indicating the calculated tensors truthfully reproduce the pseudocontact shift effects.

The determined magnetic anisotropy of Co(II) ion bound ubiquitin A28C-2V-8HQ adduct (Table 1) is close to that previously observed in the high spin cobalt(II) formed tetrahedral coordination complex with blue copper protein, azurin (Donaire et al 1998). The $\Delta \chi$-tensor magnitudes and structural feature of the cobalt complex in A28C-2V8HQ are also similar to those observed in the cobalt coordinated to the bipyridylalanine encoded West Nile virus NS2B-NS3 protease (Nguyen et al 2011), where the cobalt binding is assisted by additional protein sidechains.

The $\mathrm{Co}^{2+}$ complex of E24H/A28C-2V-8HQ adduct generates larger PCSs than that of ubiquitin A28C-2V-8HQ, and correspondingly the determined $\Delta \chi$-tensors are also larger (Table 1). More importantly, the two cobalt complexes produce different $\Delta \chi$-tensors both in magnitudes and orientations. This confirms that the single point mutation of E24H is effective to change the paramagnetic tensor significantly, suggesting different coordinating modes of Co(II) complexes in these two protein adducts. From structure modeling of the tag based on the PCS data it is evident that imidazole of histidine is coordinated to cobalt in the E24H/A28C-2V-8HQ conjugate (Table 1 and Fig. 5B).

Error-analysis of paramagnetic tensors were performed by random selection of 80 percent of the PCSs data to fit the crystal structure (Ramage et al 1994; PDB code: 1UBI) and RDC-refined NMR structure (Maltsev et al 2014; PDB code: 2MJB), respectively. Both A28C-2V-8HQ and E24H/A28C-2V-8HQ adducts complexed with Co(II) gave high precision in tensor determinations, and the outcome of 100 calculations are shown in Fig. S8. The plots of Sanson-Flamsteed projection demonstrate that the paramagnetic tensors calculated for the two Co(II) complexes of A28C-2V-8HQ and E24H/A28C-2V-8HQ differ greatly in orientations (Fig S7). The different coordination modes of E24H imidazole and E24, D32 or Q31 sidechain carboxylate or amide are likely to cause different paramagnetic anisotropy and change the angles of the principle axes of the $\Delta \chi$-tensors. In the case of $\mathrm{T} 22 \mathrm{C}-2 \mathrm{~V}-8 \mathrm{HQ}$, the determined paramagnetic tensors are more spread, suggesting the paramagnetic tensors are less reliable. This is because in the T22C-2V-8HQ conjugate the combinational coordination of 8HQ and protein sidechain (probably E24) to metal ion is ill-matched in restricting the flexibility of paramagnetic center, resulting in a loosely coordinated 
metal complex. In the structure of ubiquitin, residue Ala28 resides in the middle of the first helix and E24 and D32 are two acidic residues that reside in the $\mathrm{i}-4$ and $\mathrm{i}+4$ positions, respectively. In addition $\mathrm{Q} 31$ is located at the $\mathrm{i}+3$ position. In the case of A28C-2V-8HQ conjugate, the calculated paramagnetic center has a distance of $5.4 \AA$ to the sidechain oxygen of D32 but with a shorter distance ( 3.0 $\AA$ ) to the sidechain oxygen of Q31. However, the cobalt ion is closer to the side-chain of residue E24 with a distance of $5.0 \AA$ in the complex with E24H/A28C-2V-8HQ. In both protein conjugates, the Q-factors are very small (Fig S6). These results indicate that Q31 and E24H are involved in coordinating to cobalt (II) ion in the A28C-2V-8HQ and E24H-2V-8HQ adducts, respectively.

To obtain a structural view of the metal complex with respect to the protein, explicit tag coordinates were calculated using PyParaTool (Stanton-Cook et al). For the cobalt complex of A28C-2V-8HQ, the determined metal position resides close to the sidechain oxygen of Q31, with a distance of $3.0 \AA$, suggesting Q31 instead of D32 is coordinated to the cobalt ion in addition to 8HQ (Fig 5). In the structure of E24H/A28C-2V-8HQ, the sidechain of histidine is more favorable to bind the cobalt ion. The calculated distance of the cobalt ion to the sidechain nitrogen of His24 is $2.4 \AA$ as shown in Fig 5B, which is in good agreement with the typical coordination distance of Co(II). These results are in agreement with a fluorescence analysis of the zinc complex. The preferential coordination of Zn(II) to histidine over carboxylate or amide sidechains results in stronger fluorescence in E24H/A28C-2V-8HQ than A28C-2V-8HQ (Fig S8). It is noted that in the T22C-2V-8HQ and A28C-2V-8HQ adducts, the pair of $i$ and $\mathrm{i}+4$, where $\mathrm{i}$ is Cys and $\mathrm{i}+4$ is Glu or Asp, is not an ideal segment to restrict the transition metal ion. However, a segment in the helix containing positions of $i$ and $i+3$ or $i-4$, where $i$ is Cys, $i+3$ is Gln or Glu and $i-4$ is His, is preferred to immobilize the 2V-8HQ tag.

Comparison of PCS and RDC measured for Co(II) complexes of ubiquitin-2V-8HQ adduct

Mobility of a paramagnetic tag generally arises from chemical bond reorientations of the metal-chelating groups and from motions of the linker between protein and paramagnetic tag. The flexibility of the tag will cause different averaging in RDCs and PCSs, because PCS are distance dependent whereas RDCs are not. RDCs are more sensitive to the tag mobility than PCSs (Shishmarev and Otting 2013). To elucidate the flexibility of the tag in ubiquitin-2V-8HQ, RDCs of protein backbone amides were measured for the Co(II) complex of ubiquitin E24H/A28C-2V-8HQ. The alignment tensor was calculated by fitting the RDCs to the crystal structure of ubiquitin (PDB code: 1UBI) using the program Module (Dosset et al 2001) (Table 2). Similar results were obtained with the NMR structure (Maltsev et al 2014). The alignment tensors determined from RDCs and back-calculated from PCSs were quite similar (Table 2). The result is consistent with the FANTEN calculation (Rinaldelli et al 2014) that can 
directly determine the $\Delta \chi$-tensor parameters from the input of RDC values (Table S1). These data confirm that $2 \mathrm{~V}$ 8HQ is a rigid cobalt binding tag, which immobilizes the paramagnetic center in a similar way as the published lanthanide binding tags 4MMDPA (Su et al 2008) and 3MDPA (Man et al 2010).

PRE analysis of Mn(II) and $\mathrm{Cu}(\mathrm{II})$ complexed with 2V-8HQ adducts

PREs are extensively used in structural biology (Clore and Iwahara 2009), but caution must be taken when interpreting PREs because transient non-specific encounters between the paramagnetic center and the protein surface can contribute significant effects, requiring an assessment of whether the presence of free metal ion in the protein sample and non-specific transient associations between the paramagnetic tag to the surface of protein. Several studies have shown that ubiquitin itself binds transition metal ions (Arena et al 2011; Falini et al 2008; Arnesano et al 2011), the PRE effects on ubiquitin-2V-8HQ conjugates were therefore assessed in this study.

The PRE effects have been evaluated by comparison of cross-peak intensity changes in ${ }^{15} \mathrm{~N}-\mathrm{HSQC}$ spectra between the diamagnetic (in the absence of metal ion) and paramagnetic samples. The ubiquitin-2V-8HQ conjugates formed complexes with $\mathrm{Mn}(\mathrm{II})$ and $\mathrm{Cu}(\mathrm{II})$ ions are in slow exchange at NMR time scale. Titration of paramagnetic ions $\mathrm{Mn}(\mathrm{II})$ and $\mathrm{Cu}(\mathrm{II})$ into the solution of ubiquitin-2V-8HQ produces different effects on protein signals. For example, formation of copper (II) complex with protein not only generates significant PREs but also sizable chemical shifts changes for some residues as shown in the ${ }^{15} \mathrm{~N}-\mathrm{HSQC}$ spectra in Fig. 4. In contrast, addition of Mn(II) into the solution of protein-2V-8HQ only produces PREs on NMR signals. In general, the residues most affected by PREs are vicinal to the mutation site of A28C, indicating that the paramagnetic center is located closely to the ligation site of A28C. Assuming Mn(II) and Cu(II) share a similar structural arrangement with Co(II) bound ubiquitin-2V-8HQ, the $\mathrm{Co}(\mathrm{II})$ position was used for $\mathrm{Mn}(\mathrm{II})$ and $\mathrm{Cu}(\mathrm{II})$ in these protein complexes. The correlations of peak intensity ratio between the paramagnetic and diamagnetic cross-peaks with respect to the amino acid sequence are shown in Fig. 6. For a better comparison, the cross-peak attenuation with respect to the distances of backbone amide protons to the paramagnetic center is also shown in Fig. 6. Amide protons within a $8 \AA$ radius of the paramagnetic center are generally broadened beyond detections in ${ }^{15} \mathrm{~N}-\mathrm{HSQC}$ spectra of A28C and E24H/A28C-2V-8HQ adducts complexed with $\mathrm{Mn}(\mathrm{II})$ and $\mathrm{Cu}(\mathrm{II})$ ions. In the case of A28C-2V-8HQ conjugate, significant peak attenuations were also observed for the residues Gln2, Ile3 and Gly76 at the N- and C-terminus, which are likely due to solvent PREs at a protein centration of $0.1 \mathrm{mM}$. In general, the peak attenuations are in good agreement with the calculated distances of amide protons to the paramagnetic center for both $\mathrm{Mn}(\mathrm{II})$ and $\mathrm{Cu}(\mathrm{II})$ complexes.

For the metal complexes of E24H/A28C-2V-8HQ, excellent correlations of PREs on amide protons with distances to the paramagnetic center were observed (Fig. 6B). Compared with A28C-2V-8HQ, the cross-peak 
attenuations determined for the $\mathrm{Mn}(\mathrm{II})$ and $\mathrm{Cu}(\mathrm{II})$ complexes with E24H/A28C-2V-8HQ are tightly related to the position of the paramagnetic center as simulated by PCSs of Co(II) complex, indicating the structural modes of $\mathrm{Mn}(\mathrm{II})$ and $\mathrm{Cu}(\mathrm{II})$ bound to histidine are more similar than to the sidechain carboxylate or amide. The last flexible C-terminal residues also experience significant PREs for the complex of $\mathrm{Mn}(\mathrm{II})$, however, the peak attenuations in the $\mathrm{Cu}(\mathrm{II})$ complex for these C-terminal residues are equally to those in the structured region. This is probably caused by solvent PREs by excess of Mn(II) during titrations. In both protein samples, no indication of free paramagnetic ion bound to the protein surfaces as shown previously (Arena et al 2011; Falini et al 2008; Arnesano et al 2011) was found.

Compared with published small paramagnetic tags, 4MMDPA (Su et al 2008), 3MDPA (Man et al 2010), 4MDPA (Jia et al 2011), NTA (Swarbrick et al 2011) and IDA tags (Loh et al 2015), which are suitable for lanthanide ions, 2V-8HQ is of similar size but has high binding affinity for transition metal ions. In addition, C-S thiol ether protein conjugates are stable and resistant to reducing reagent that can be applied in NMR analysis under reducing environment. The cobalt (II) coordinated 3MDPA protein adduct reported previously showed significantly smaller paramagnetic tensors compared with A28C-2V-8HQ and E24H/A28C-2V-8HQ, implying a different coordination of 8HQ to cobalt. As lanthanide ions generally have coordination numbers of eight to ten, small lanthanide binding tags do not easily afford strong binding capability and generally fast exchange between protein and protein-metal complex will be observed in NMR spectra, which can result in significant solvent PREs and non-specific association of free metal ion to protein surface. $2 \mathrm{~V}-8 \mathrm{HQ}$ is not suitable for lanthanide ions but its advantage lies in its strong binding affinity for transition metal ions and fluorescent property in complex with zinc (II), which can be used in fluorescent energy transfer (FRET) measurements. Due to its small size and rigidity, 2V-8HQ is a useful paramagnetic and fluorescent tag that can be used in structural biology by NMR and fluorescence analysis. With advent of the chemically synthesized tags, selective modification of one thiol group can possibly be made for a protein with two or more cysteines, provided that the cysteines have different solvent accessibilities and local mobilities as those effects will greatly affect the reactivity of thiols (Ma et al 2014).

\section{Conclusions}

We show that 8HQ, a well-known transition metal chelator, can be site-specifically attached to a protein in a Michael addition-like thiol-ene reaction. The protein-2V-8HQ conjugates contain a stable thiol ether tether and show high binding affinities with transition metal ions. The determined paramagnetic tensors of cobalt possess the character of high-spin cobalt (II) complexes. The metal binding affinity can be further enhanced in a helix (Yagi et al 2013), if $i$ is histidine and $i+4$ the tagged cysteine. Different paramagnetic tensors can simply be achieved by 
changing the coordination of the paramagnetic ion by amino acid sidechains in 2V-8HQ labeled protein. In summary, 2V-8HQ offers an attractive way of coordinating transition metal ions, producing a rigid, stable and small transition metal binding tag that can be used in structural analysis by NMR and also fluorescence spectroscopy.

\section{Acknowledgement}

Financial support by the 973 program (2013CB910200), the National Science Foundation of China (21473095 and 21273121), and the Australian Research Council (DP120100561 and DP150100383) is greatly acknowledged.

\section{References}

Abragam A, Bleaney B (1970) Electron paramagnetic resonance of transition ions. Oxford University Press, Oxford

Alacid E, Najera C (2008) Aqueous Sodium Hydroxide Promoted Cross-Coupling Reactions of Alkenyltrialkoxysilanes under Ligand-Free Conditions. J Org Chem 73:2315-2322

Arena G, Fattorusso R, Grasso G, Grasso GI, Isernia C, Malgieri G, Milardi D, Rizzarelli E (2011). Zinc(II) complexes of ubiquitin: speciation, affinity and binding features. Chem Eur J 17:11596-11603

Arnesano F, Banci L, Bertini I, Felli IC, Luchinat C, Thompsett AR (2003) A strategy for the NMR characterization of type II copper(II) proteins: the case of the copper trafficking protein CopC from Pseudomonas Syringae. J Am Chem Soc 125:7200-7208

Arnesano F, Banci L, Piccoli M (2005) NMR structures of paramagnetic metalloproteins. Q Rev Biophys 38:167219

Arnesano F, Belviso BD, Caliandro R, Falini G, Fermani S, Natile G, Siliqi D (2011) Crystallographic analysis of metal-ion binding to human ubiquitin. Chem Eur J 17:1569-1678

Bertini I, Luchinat C (1996) NMR of paramagnetic substances. Coord Chem Rev 150:1-243

Bertini I, Luchinat C (1984) High spin cobalt(II) as a probe for the investigation of metalloproteins. Adv Inorg Biochem 6:71-111

Bertini I, Luchinat C (1999) New applications of paramagnetic NMR in chemical biology. Curr Opin Chem Biol 3:145-151 
Bertini I, Luchinat C, Parigi G (2002) Magnetic susceptibility in paramagnetic NMR. Prog NMR Spectr 40:249273

Bertini I, Luchinat C, Parigi G, Pierattelli R (2005) NMR spectroscopy of paramagnetic metalloproteins. Chembiochem 6:1536-1549

Bertini I, Luchinat C, Parigi G, Pierattelli R (2008) Perspectives in paramagnetic NMR of metalloproteins. Dalton:3782-3790

Bertini I, Turano P, Vila AJ (1993) Nuclear magnetic resonance of metalloproteins. Chem Rev 93:2833-2932

Cao C, Chen JL, Yang Y, Huang F, Otting G, Su XC (2014) Selective ${ }^{15} \mathrm{~N}$-labeling of the side-chain amide groups of asparagine and glutamine for applications in paramagnetic NMR spectroscopy. J Biomol NMR 59:251261

Clore GM, Iwahara J (2009) Theory, practice, and applications of paramagnetic relaxation enhancement for the characterization of transient low-population states of biological macromolecules and their complexes. Chem Rev 109:4108-4139

Donaire A, Salgado J, Moratal JM (1998) Determination of the magnetic axes of cobalt(II) and nickel(II) azurins from ${ }^{1} \mathrm{H}$ NMR data: influence of the metal and axial ligands on the origin of magnetic anisotropy in blue copper proteins. Biochemistry 37:8659-8673

Dosset P, Hus JC, Marion D, Blackledge M (2001) A novel interactive tool for rigid-body modeling of multi-domain macromolecules using residual dipolar couplings. J Biomol NMR 20: 223-231

Falini G, Fermani S, Tosi G, Arnesano F, Natile G (2008) Structural probing of Zn(II), Cd(II) and Hg(II) binding to human ubiquitin. Chem Commun:5980-5962

Geraldes CF (1993) Lanthanide shift reagents. Methods Enzymol 227:43-78

Geraldes CF, Luchinat C (2003) Lanthanides as shift and relaxation agents in elucidating the structure of proteins and nucleic acids. Met Ions Biol Syst 40: 513-588 
Gochin M (1998) Nuclear magnetic resonance characterization of a paramagnetic DNA-drug complex with high spin cobalt; assignment of the ${ }^{1} \mathrm{H}$ and ${ }^{31} \mathrm{P}$ NMR spectra, and determination of electronic, spectroscopic and molecular properties. J Biomol NMR 12:243-257

Koehler J, Meiler J (2011) Expanding the utility of NMR restraints with paramagnetic compounds: background and practical aspects. Prog NMR Spectr 59: 360-389

Huang F, Pei YY, Zuo HH, Chen JL, Yang Y, Su XC (2013) Bioconjugation of proteins with a paramagnetic NMR and fluorescent tag. Chem Eur J 19:17141-17149

Jensen MR, Lauritzen C, Dahl SW, Pedersen J, Led JJ (2004) Binding ability of a HHP-tagged protein towards $\mathrm{Ni}^{2+}$ studied by paramagnetic NMR relaxation: the possibility of obtaining long-range structure information. $\mathrm{J}$ Biomol NMR 29:175-185

Jensen MR, Led JJ (2006) Metal-protein interactions: structure information from Ni(II)-induced pseudocontact shifts in a native nonmetalloprotein. Biochemistry 45:8782-8787.

Jia X, Maleckis A, Huber T, Otting G (2011) 4,4'-dithiobisdipicolinic acid: a small and convenient lanthanide binding tag for protein NMR spectroscopy. Chem Eur J 17: 6830-6836

Johnston WD, Freiser H (1952) Structure and Behavior of Organic Analytical Reagents. III. Stability of Chelates of 8-Hydroxyquinoline and Analogous Reagents. J Am Chem Soc 74:5239-5242

Jones CE, Klewpatinond M, Abdelraheim SR, Brown DR, Viles JH (2005). Probing copper binding to the prion protein using diamagnetic nickel and 1H NMR: the unstructured $\mathrm{N}$ terminus facilitates the coordination of six copper ions at physiological concentrations. J Mol Biol 346:1393-1407

La Mar GN, Horrocks WD, Holm RH. (1973) NMR of paramagnetic molecules. Elsevier, Amsterdam

Lee HS, Spraggon G, Schultz PG, Wang F (2009) Genetic incorporation of a metal-ion chelating amino acid into proteins as a biophysical probe. J Am Chem Soc 131:2481-2483

Li QF, Yang Y, Maleckis A, Otting G, Su XC (2012) Thiol-ene reaction: a versatile tool in site-specific labelling of proteins with chemically inert tags for paramagnetic NMR. Chem Commun 48:2704-2706 
Liu X, Li J, Hu C, Zhou Q, Zhang W, Hu M, Zhou J, Wang J (2013). Significant expansion of the fluorescent protein chromophore through the genetic incorporation of a metal-chelating unnatural amino acid. Angew Chem Int Ed Engl 52:4805-4809

Liu WM, Overhand M, Ubbink M (2014) The application of paramagnetic lanthanoid ions in NMR spectroscopy on proteins. Coord Chem Rev 273-274: 2-12

Loh CT, Graham B, Abdelkader EH, Tuck KL, Otting G (2015) Generation of pseudocontact shifts in proteins with lanthanides using small "clickable" nitrilotriacetic acid and iminodiacetic acid tags. Chem Eur J 21:50845092

Ma FH, Chen JL, Li QF, Zuo HH, Huang F, Su XC (2014) Kinetic assay of the Michael addition-like thiol-ene reaction and insight into protein bioconjugation. Chem Asian J 9:1808-1816

Man B, Su XC, Liang H, Simonsen S, Huber T, Messerle BA, Otting G (2010) 3-Mercapto-2,6-pyridinedicarboxylic acid: a small lanthanide-binding tag for protein studies by NMR spectroscopy. Chem Eur J 16:3827-3832.

Maltsev AS, Grishaev A, Roche J, Zasloff M, Bax A (2014) Improved cross validation of a static ubiquitin structure derived from high precision residual dipolar couplings measured in a drug-based liquid crystalline phase. J Am Chem Soc 136:3752-3755

Marley J, Lu M, Bracken C (2001) A method for efficient isotopic labeling of recombinant proteins. J Biomol NMR 20:71-75

Nguyen THD, Ozawa K, Stanton-Cook M, Barrow R, Huber T, Otting G (2011) Generation of pseudocontact shifts in protein NMR spectra with a genetically encoded cobalt(II)-binding amino acid. Angew Chem Int Ed 50:692-694

Otting G (2008) Prospects for the lanthanides in structural biology by NMR. J Biomol NMR 42: 1-9

Otting G (2010) Protein NMR using paramagnetic ions. Annu Rev Biophys 39: 387-405

Park SH, Wang VS, Radoicic J, De Angelis AA, Berkamp S, Opella SJ (2015). Paramagnetic relaxation enhancement of membrane proteins by incorporation of the metal-chelating unnatural amino acid 2-amino-3-(8- 
hydroxyquinolin-3-yl)propanoic acid (HQA) J Biomol NMR 61:185-196

Petitjean A, Kyritsakas N, Lehn JM (2005) Ion-Triggered Multistate Molecular Switching Device Based on Regioselective Coordination-Controlled Ion Binding. Chem Eur J 11: 6818-6828.

Pintacuda G, John M, Su XC, Otting G (2007) NMR structure determination of protein-ligand complexes by lanthanide labelling. Acc Chem Res 40:206-212

Qi A, Gross A, Jeschke G, Godt A, Drescher M (2014) Gd(III)-PyMTA label is suitable for in-cell EPR. J Am Chem Soc 136:15366-15378

Ramage R, Green J, Muir TW, Ogunjobi OM, Love S, Shaw K (1994) Synthetic, structural and biological studies of the ubiquitin system: chemically synthesized and native ubiquitin fold into identical three-dimensional structures. Biochem J 299:151-158

Rinaldelli M, Carlon A, Ravera E, Parigi G, Luchinat C (2014) FANTEN: a new web-based interface for the analysis of magnetic anisotropy-induced NMR data. J Biomol NMR 61:21-34

Rodriguez-Castañeda F, Haberz P, Leonov A, Griesinger C (2006) Paramagnetic tagging of diamagnetic proteins for solution NMR. Magn Reson Chem 44:S10-16

Shishmarev D, Otting G (2013) How reliable are pseudocontact shifts induced in proteins and ligands by mobile paramagnetic metal tags? A modelling study J Biomol NMR 56:203-216

Schmitz C, Stanton-Cook MJ, Su XC, Otting G, Huber T (2008) Numbat: an interactive software tool for fitting $\Delta \chi$ tensors to molecular coordinates using pseudocontact shifts. J Biomol NMR 41:179-189

Stanton-Cook MJ, Su XC, Otting G, Huber T, http://compbio.anu.edu.au/mscook/PPT/

Su XC, Man B, Beeren S, Liang H, Simonsen S, Schmitz C, Huber T, Messerle BA, Otting G (2008) A dipicolinic acid tag for rigid lanthanide tagging of proteins and paramagnetic NMR spectroscopy. J Am Chem Soc 130:10486-10487

Swarbrick JD, Ung P, Su XC, Maleckis A, Chhabra S, Huber T, Otting G, Graham B (2011) Engineering of a bischelator motif into a protein $\alpha$-helix for rigid lanthanide binding and paramagnetic NMR spectroscopy. Chem 
Commun (Camb) 47:7368-7370

Yagi H, Maleckis A, Otting G (2013). A systematic study of labelling an $\alpha$-helix in a protein with a lanthanide using IDA-SH or NTA-SH tags. J Biomol NMR 55:157-166

Yang Y, Li QF, Cao C, Huang F, Su XC (2013) Site-specific labeling of proteins with a chemically stable, highaffinity tag for protein study. Chem Eur J 19:1097-1103 
Table 1. $\Delta \chi$-tensor parameters of ubiquitin-2V-8HQ complexes with Co(II) ion. ${ }^{[\mathrm{a}]}$

\begin{tabular}{llll}
\hline & A28C & E24H/A28C & T22C \\
\hline$\Delta \chi_{\mathrm{ax}}$ & $8.3(7.5)$ & $-10.3(-8.6)$ & $-2.1(-2.2)$ \\
$\Delta \chi_{\mathrm{rh}}$ & $5.0(4.4)$ & $-3.7(-2.8)$ & $-0.6(-0.8)$ \\
$\alpha$ & $64.1(117.3)$ & $79.4(56.9)$ & $90.6(134.8)$ \\
$\beta$ & $23.7(92.3)$ & $141.2(90.1)$ & $35.7(78.3)$ \\
$\gamma$ & $91.2(167.8)$ & $66.5(31.9)$ & $85.5(174.6)$ \\
\hline
\end{tabular}

a The tensor parameters are in units of $10^{-32} \mathrm{~m}^{3}$ and were determined by fitting the PCS data of backbone amide protons to the crystal structure of ubiquitin (PDB code: 1UBI). For comparison, the tensor parameters by using the RDC refined ubiquitin structure (PDB code: 2MJB, first conformer) are shown in bracket.

Table 2. Comparison of alignment tensor and $\Delta \chi$-tensor parameters of ubiquitin E24H/A28C-2V8HQ complexed with Co(II) ion. ${ }^{\mathrm{a}}$

\begin{tabular}{lllll}
\hline & $10^{4} A_{\mathrm{ax}}{ }^{\mathrm{b}}$ & $10^{4} A_{\mathrm{rh}}{ }^{\mathrm{b}}$ & $10^{4} A_{\mathrm{ax}}{ }^{\mathrm{c}}$ & $10^{4} A_{\mathrm{rh}}{ }^{\mathrm{c}}$ \\
\hline $\mathrm{Co}(\mathrm{II})$ & 2.2 & 1.5 & $2.6(2.2)$ & $0.9(0.7)$ \\
\hline
\end{tabular}

${ }^{a}$ All data recorded at $25^{\circ} \mathrm{C}$ and $600 \mathrm{MHz}{ }^{1} \mathrm{H}$ NMR frequency.

b Alignment tensor determined using Module (Dosset et al. 2001). Only RDCs of residues in regular secondary structure elements were used in the fit (residues 2-8, 12-17, 41-45, 47-50, 65-70).

${ }^{\mathrm{c}}$ Alignment tensor predicated from the $\Delta \chi$-tensor values of Table 1 using

$A_{a x, r h}=\frac{B_{0}^{2}}{15 k T \mu_{0}} \Delta \chi_{a x, r h}$ 
Figure Legend

Fig. 1 Molecular structure of 2V-8HQ.

Fig. 2 A) Superimposition of ${ }^{15} \mathrm{~N}-\mathrm{HSQC}$ spectra of a $0.1 \mathrm{mM}$ solution of uniformly ${ }^{15} \mathrm{~N}$-labeled ubiquitin E24H/A28C ubiquitin (black) and E24H/A28C-2V-8HQ conjugate (red). B) Chemical-shift differences between free protein and its 2V-8HQ conjugate, calculated as $\Delta \delta=\left(\left(\Delta \delta_{\mathrm{H}}\right)^{2}+\left(\Delta \delta_{\mathrm{N}} / 10\right)^{2}\right)^{1 / 2}$ : T22C (red circle); A28C (blue triangle); E24H/A28C (black square). It is noted that significant chemical shift changes were also observed for residues 50-60 that are close to the labelling site T22.

Fig. 3 A) Superimposition of ${ }^{15} \mathrm{~N}$-HSQC spectra of a $0.1 \mathrm{mM}$ solution of uniformly ${ }^{15} \mathrm{~N}$-labeled ubiquitin E24H/A28C-2V-8HQ in the presence of one equivalent Zn(II) (black) and one equivalent Co(II) (red), respectively. B) PCSs of protein backbone amide protons generated by Co(II) in complex with ubiquitin 2V-8HQ adducts of T22C (solid circle), A28C (triangle), E24H/A28C (square). The PCSs were calculated by chemical shift differences between paramagnetic and diamagnetic ( $\mathrm{Zn}(\mathrm{II})$ as the reference) samples.

Fig. 4 Superimposition of ${ }^{15} \mathrm{~N}-\mathrm{HSQC}$ spectra of ubiquitin-2V-8HQ (red) complexed with Mn(II) and Cu(II) (black), respectively. A) $0.1 \mathrm{mM}$ A28C-2V-8HQ complexed with $0.1 \mathrm{mM} \mathrm{Mn(II)}$ and $\mathrm{Cu}(\mathrm{II})$, respectively; B) $0.05 \mathrm{mM}$ E24H/A28C-2V-8HQ complexed with $0.05 \mathrm{mM} \mathrm{Mn(II)} \mathrm{and} \mathrm{Cu(II),} \mathrm{respectively.} \mathrm{NMR} \mathrm{conditions} \mathrm{were} \mathrm{the} \mathrm{same} \mathrm{as}$ shown in experimental section.

Fig. 5 Structural view of ubiquitin-2V-8HQ complexed with Co(II) ion. A) A28C-2V-8HQ; B) E24H/A28C-2V8HQ. The calculated paramagnetic center was labeled with sphere (magenta) and the distance of metal ion to the sidechain amide oxygen and nitrogen of imidazole was connected with solid line, respectively. The structure was determined by PyParaTool.

Fig. 6 PREs analysis of protein backbone amide protons calculated by peak intensity ratios in ${ }^{15} \mathrm{~N}-\mathrm{HSQC}$ spectra for the protein samples in the presence and absence of one equivalent paramagntic ion $\mathrm{Mn}(\mathrm{II})$ (circle) or $\mathrm{Cu}(\mathrm{II})$ (triangle). A) $0.1 \mathrm{mM}$ A28C-2V-8HQ; B) $0.05 \mathrm{mM} \mathrm{E24H/A28C-2V-8HQ.} \mathrm{The} \mathrm{peak} \mathrm{intensities} \mathrm{between} \mathrm{I}_{\text {para }} / \mathrm{I}_{\text {dia }}\left(\mathrm{I}_{\text {para }}\right.$ and $\mathrm{I}_{\text {dia }}$ depict the cross peak intensites for the residues in the presence and absence of paramagnetic metal ion, respectively) were shown in left and the calculated distances (shown in line) of paramagnetic center (determined by fitting PCSs data to the crystal structure of ubiquitin (PDB code:1UBI)) and the backbone amide protons were shown in right axes, respectively. 



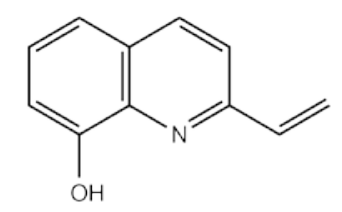

Fig. 1 

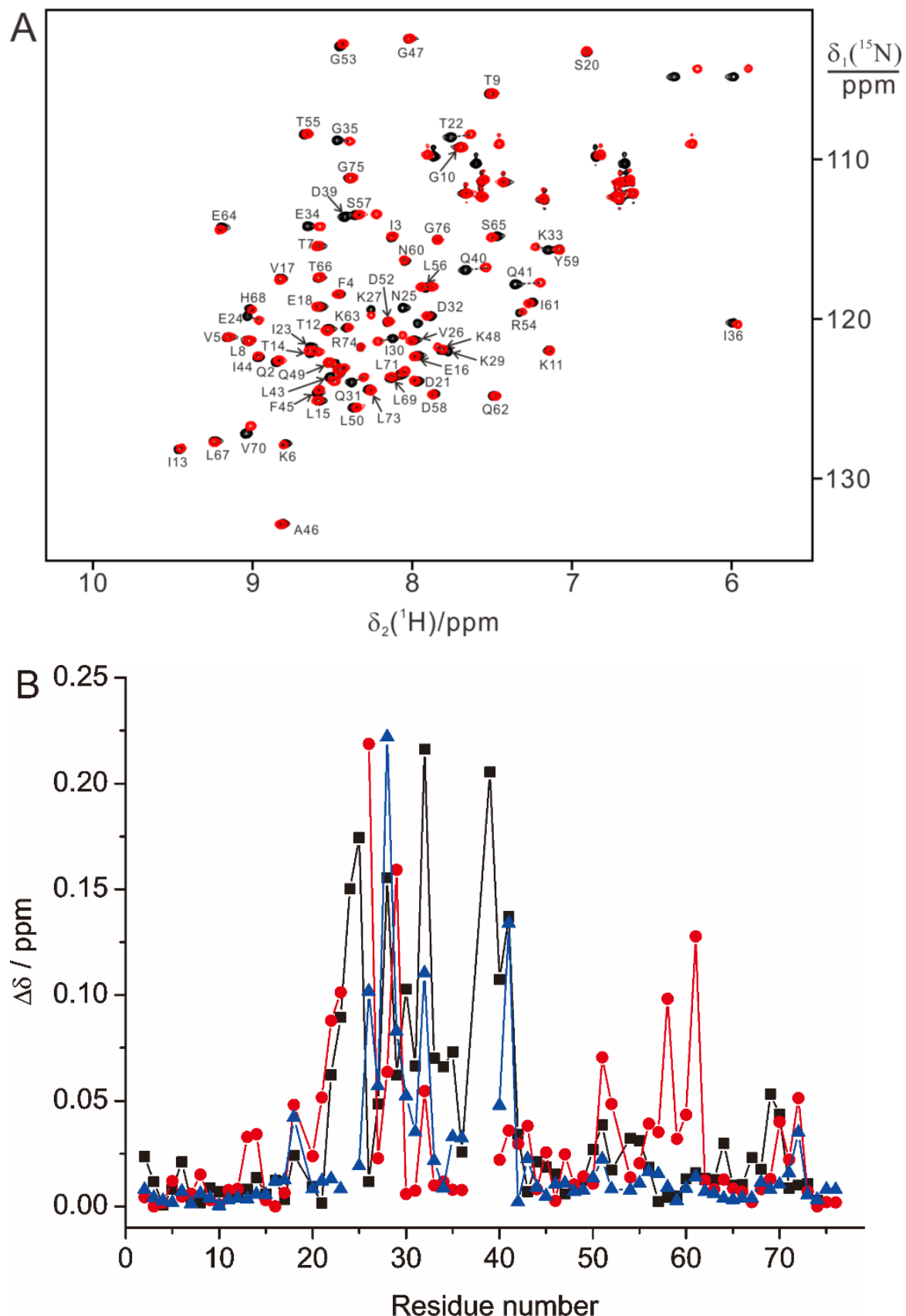

Fig. 2 


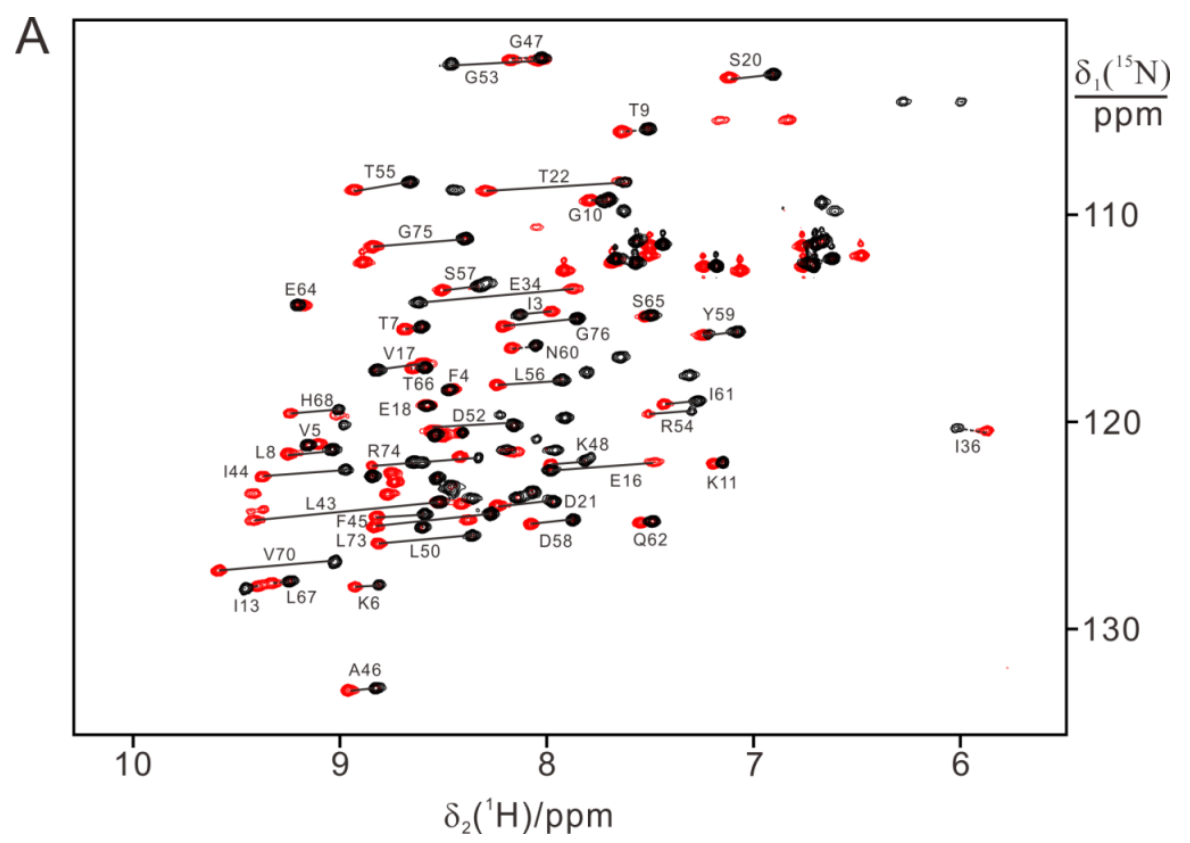

B

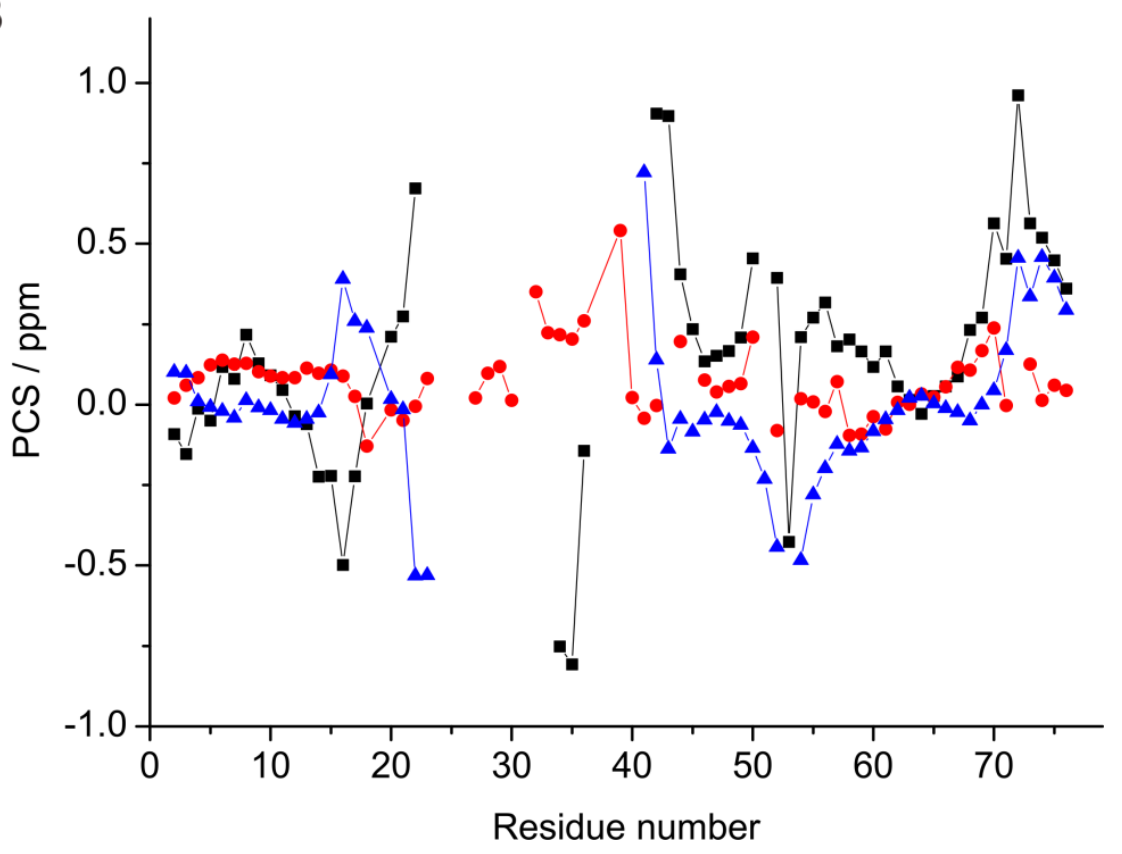

Fig. 3 

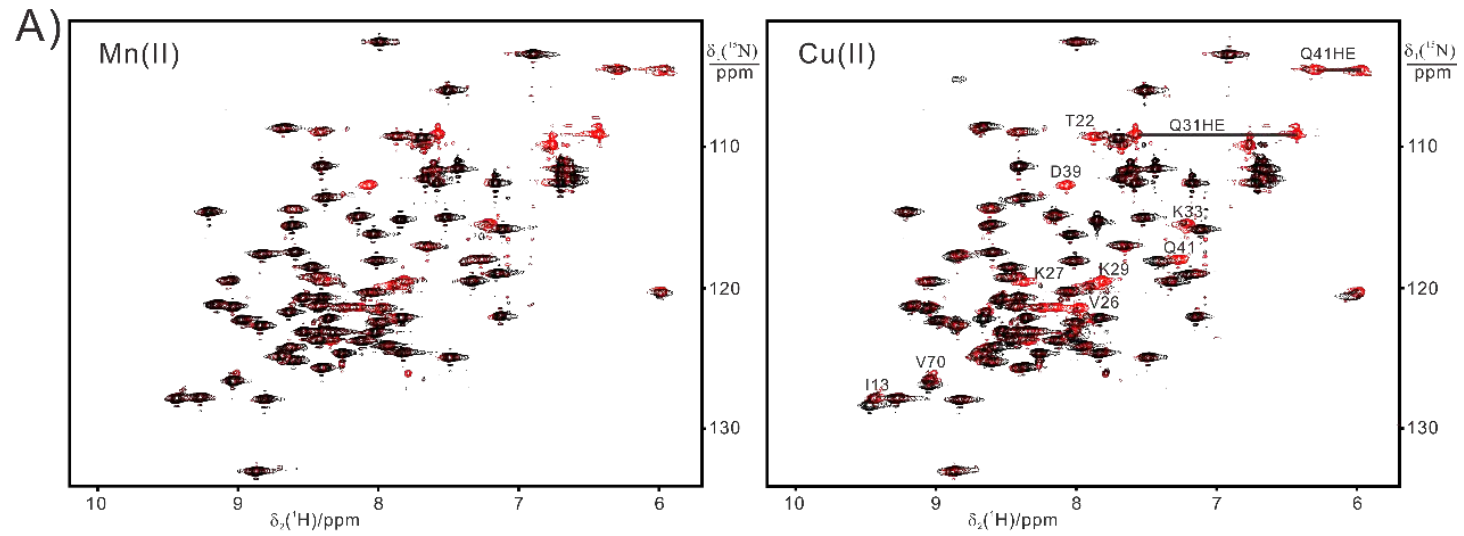

B)
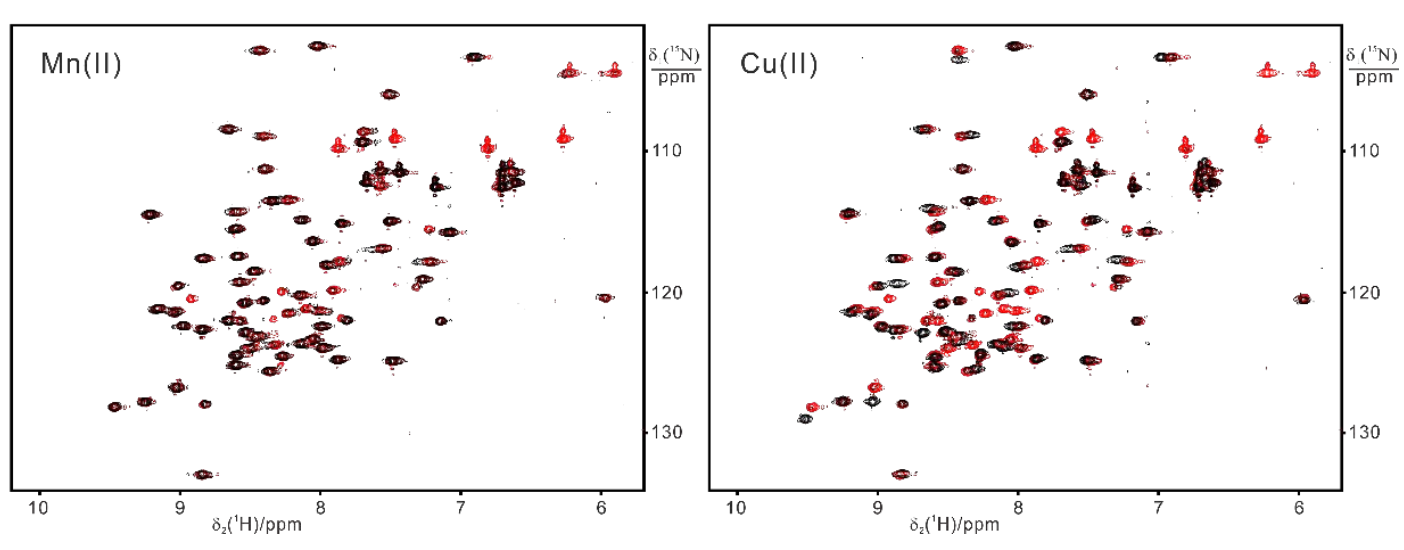

Fig. 4
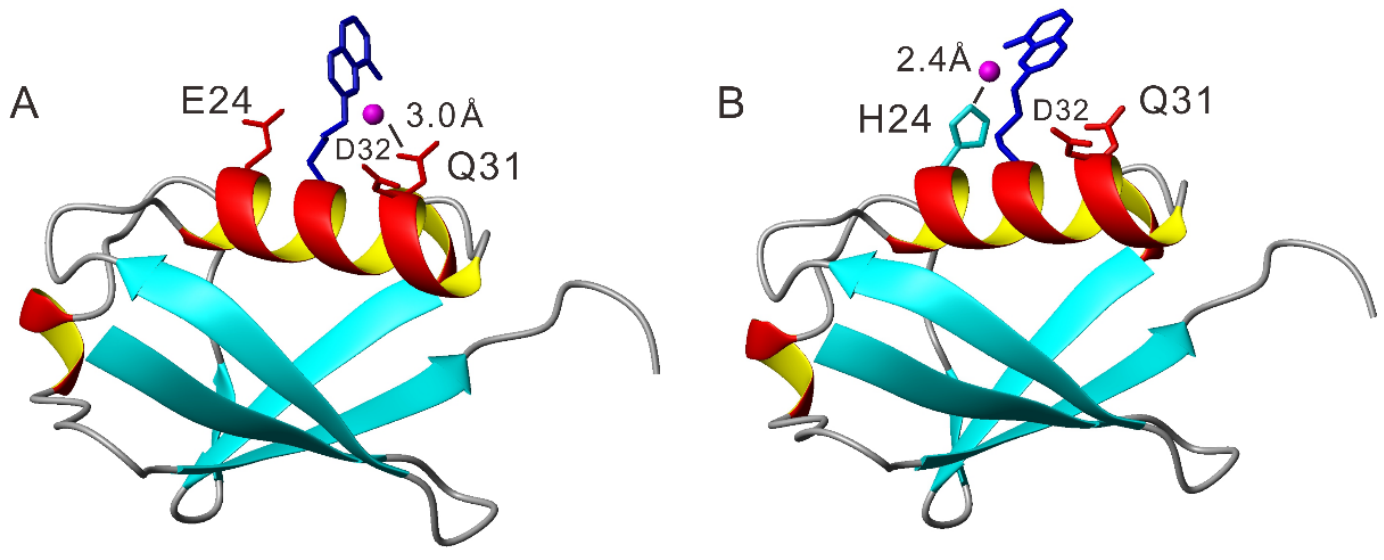

Fig. 5 

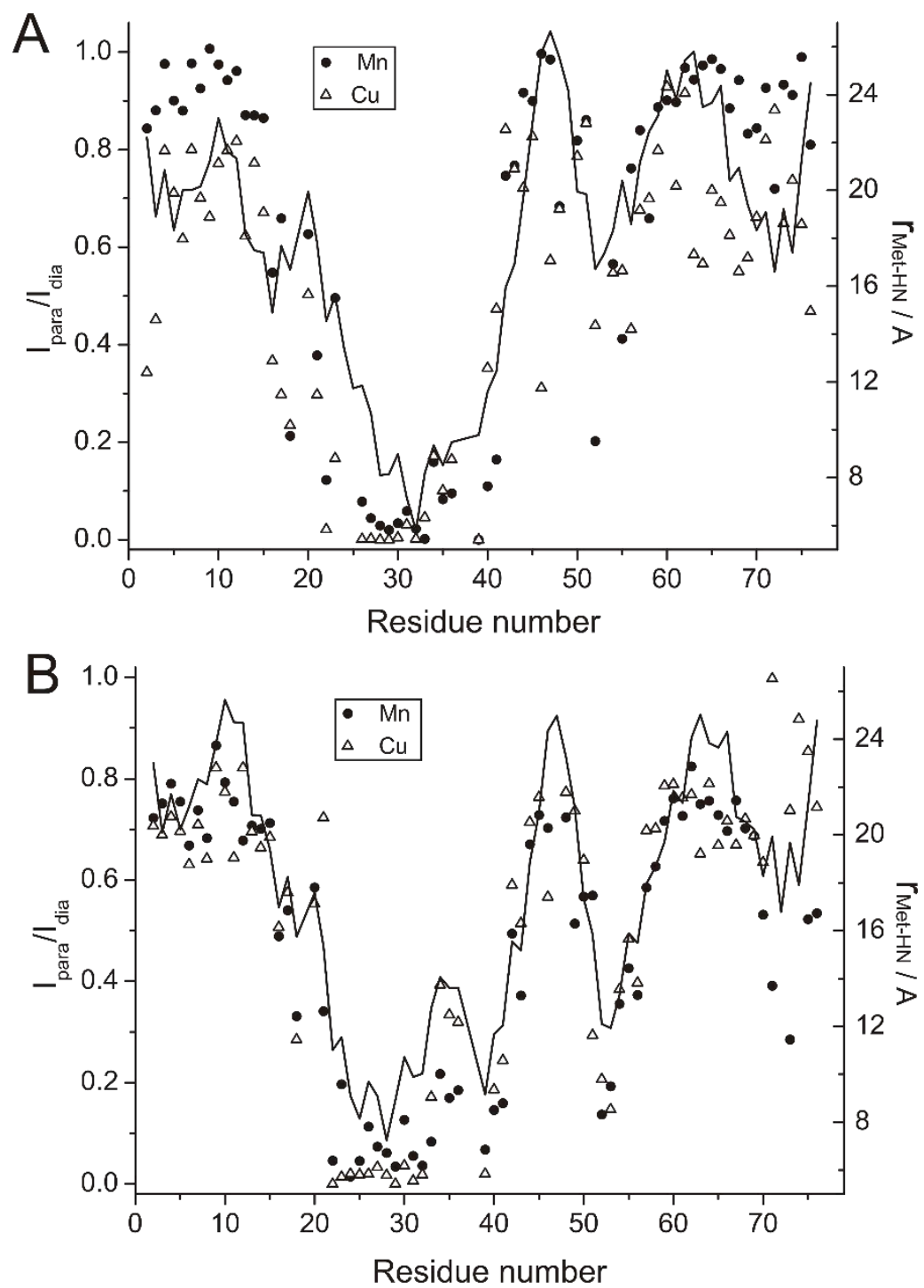

Fig. 6 